\title{
Intermolecular Headgroup Interaction and Hydration as Driving Forces for Lipid Transmembrane Asymmetry
}

\author{
Nikolay Smolentseva ${ }^{a}$, Cornelis Lütgebaucks ${ }^{a}$, Halil I. Okur, Alex G. F. de Beer and Sylvie Roke* \\ Laboratory for fundamental BioPhotonics (LBP), Institute of Bioengineering (IBI), and Institute of Materials Science (IMX), \\ School of Engineering (STI), and Lausanne Centre for Ultrafast Science (LACUS), École Polytechnique Fédérale de \\ Lausanne (EPFL), CH-1015 Lausanne, Switzerland \\ a These two authors contributed equally \\ * Corresponding author: sylvie.roke@epfl.ch
}

This PDF file includes:

Supplementary text

S1. Lipids - Structure and liposome characterization

S2. Normalization and correction for the difference in size distribution

S3. Calculation of an effective radius

S4. SFS spectral fitting

S5. Calculation of the orientational distribution of phosphate groups

S6. Calculation of the degree of asymmetry based on geometrical arguments

S7. Calculation of the degree of asymmetry from the SFS data

S8. Additional SFS experiments

References

Figures S1 to S2

Tables S1 to S5 


\section{Supplementary Text}

\section{S1. Lipids - Structure and liposome characterization}

Lipids used in this study are: 1,2-dioleoyl-sn-glycero-3-phosphocholine (DOPC), 1,2-dipalmitoylsn-glycero-3-phosphocholine (DPPC), 1,2-dioleoyl-sn-glycero-3-phospho-L-serine (sodium salt) (DOPS), 1,2-dipalmitoyl-sn-glycero-3-phospho-L-serine (sodium salt) (DPPS), 1,2-dipalmitoyl-snglycero-3-phosphate (sodium salt) (DPPA), 1,2-dipalmitoyl-sn-glycero-3-phosphoethanolamine (DPPE), $\mathrm{d}_{62}$-1,2-dipalmitoyl-sn-glycero-3-phospho-L-serine (sodium salt) ( $\mathrm{d}_{62}$-DPPS), and $\mathrm{d}_{66}-1,2-$ dioleoyl-sn-glycero-3-phosphocholine $\left(\mathrm{d}_{66}\right.$-DOPC). The chemical structures of the used lipids are presented in Fig. S1 and the liposome characterization is given in Table S1.

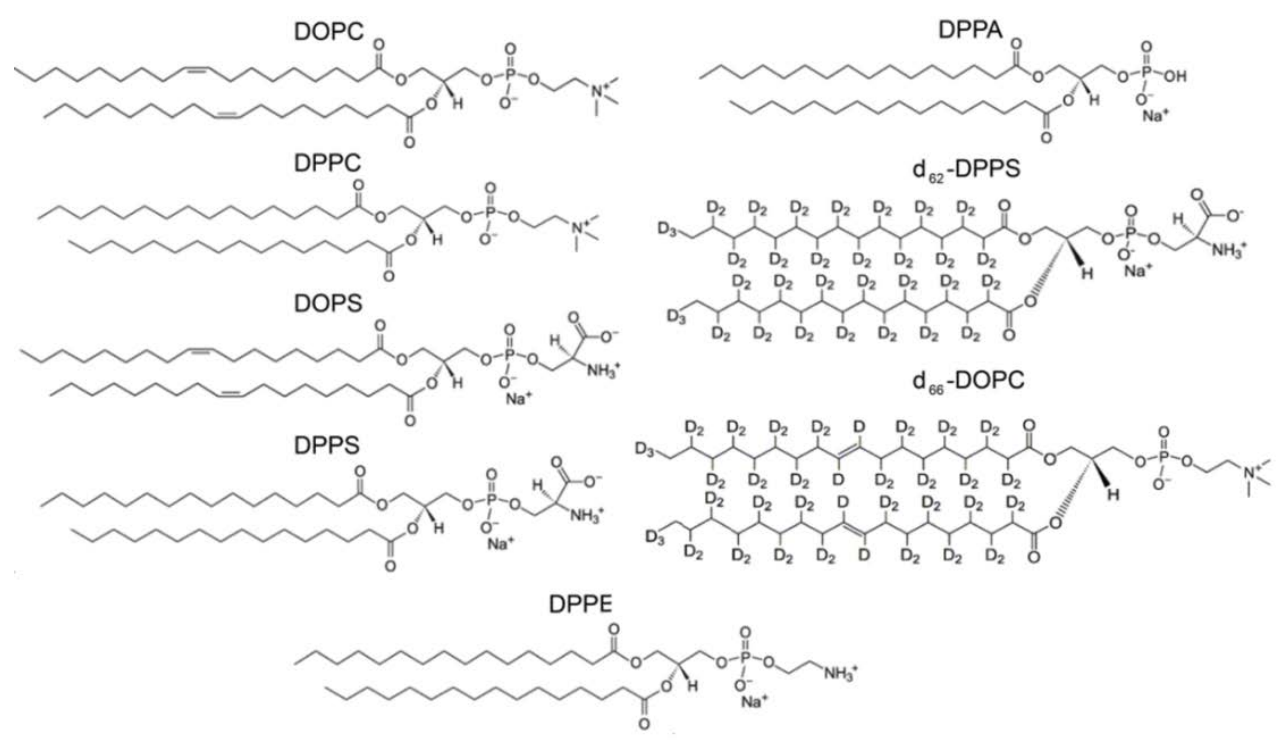

Figure S1: Chemical structures of the used phospholipids.

Table S1. The results of dynamic light scattering and zeta potential measurements. Standard deviations from the mean of three measurement are given in parenthesis.

\begin{tabular}{|c|c|c|}
\hline Sample & Hydrodynamic diameter $(\mathrm{nm})$ & Zeta potential $(\mathrm{mV})$ \\
\hline DPPS & $98.4(0.5)$ & $-45(1)$ \\
\hline DOPC & $94.0(0.3)$ & $-6(1)$ \\
\hline DPPC & $96.0(0.3)$ & $-4(1)$ \\
\hline $\mathrm{d}_{66}$-DOPC:DPPS & $90.4(0.4)$ & $-42(1)$ \\
\hline DOPC: $\mathrm{d}_{62}$-DPPS & $69.8(0.3)$ & $-43(1)$ \\
\hline DOPC:DPPS & $95.3(0.3)$ & $-43(1)$ \\
\hline $\mathrm{d}_{66}$-DOPC:DPPA & $99.2(0.5)$ & $-36(1)$ \\
\hline DOPS:DPPS & $73.0(0.3)$ & $-52(2)$ \\
\hline
\end{tabular}


Vibrational sum frequency spectra were measured using the setup for sum frequency generation experiments described in Ref. ${ }^{1-3}$. An $800 \mathrm{~nm}$ regeneratively amplified Ti:sapphire system (Spitfire Pro, Spectra physics) seeded with an $80 \mathrm{MHz} 800 \mathrm{~nm}$ oscillator (Integral 50, Femtolasers) was operated at a $1 \mathrm{kHz}$ repetition rate to pump a commercial OPG/OPA/DPG system (HE-TOPAS-C, Light Conversion), which was used to generate IR pulses. The visible beam was split off directly from the amplifier, and spectrally shaped with a home-built pulse shaper. The angle between the $10 \mu \mathrm{J}$ visible (VIS) beam (800 nm, FWHM $\left.15 \mathrm{~cm}^{-1}\right)$ and the $6 \mu \mathrm{J} \mathrm{IR}$ beam (9700 nm or $3200 \mathrm{~nm}$, FWHM $160 \mathrm{~cm}^{-1}$ ) was $20^{\circ}$ (as measured in air). The focused laser beams were overlapped in a sample cuvette with a path length of $200 \mu \mathrm{m}$. At a scattering angle of $55^{\circ}$, the scattered SF light was collimated using a plano-convex lens ( $f=15 \mathrm{~mm}$, Thorlabs LA1540-B) and passed through two short wave pass filters (3rd Millenium, 3RD770SP). The SF light was spectrally dispersed with a monochromator (Acton, SpectraPro 2300i) and detected with an intensified CCD camera (Princeton Instruments, PI-Max3) using a gate width of $10 \mathrm{~ns}$. The acquisition time for a single spectrum was 10-20 min for $\mathrm{PO}$ stretch modes and $40 \mathrm{~min}$ for $\mathrm{CH}$ stretch modes for liposomes. A Glan-Taylor prism (Thorlabs, GT15-B), a half-wave plate (EKSMA, 460-4215) and a polarizing beam splitter cube (CVI, PBS-800-050) and two $\mathrm{BaF}_{2}$ wire grid polarizers (Thorlabs, WP25H-B) were used to control the polarization of the SFG, VIS and IR beams respectively. The SFG, and VIS beams were polarized in the vertical (S) direction, and the IR beam was polarized in the horizontal plane $(P)$, leading to the polarization combination $S_{\text {out }} S_{i n} P_{\text {in }}$. The recorded intensity was baseline subtracted and normalized to the SFG spectrum of a gold mirror in $P_{\text {out }} P_{\text {in }} P_{\text {in }}$ polarization that was recorded before each measurement.

Second harmonic scattering measurements were performed, as previously described in detail $^{4}$, using $190 \mathrm{fs}$ laser pulses at $1028 \mathrm{~nm}$ with a $200 \mathrm{kHz}$ repetition rate. The input polarization is controlled by a Glan-Taylor polarizer (GT10-B, Thorlabs) and a zero-order half wave plate (WPH05M-1030). The filtered (FEL0750, Thorlabs) input pulses with a pulse energy of $0.3 \mu \mathrm{J}$ (incident laser power $\mathrm{P}=60 \mathrm{~mW}$ ) were focused into a cylindrical glass sample cell $(4.2 \mathrm{~mm}$ inner diameter) with a waist diameter of $\sim 32 \mu \mathrm{m}$ and a Rayleigh length of $3.2 \mathrm{~mm}$. The scattered SH light was collected and collimated with a plano-convex lens $(f=5 \mathrm{~cm}$ ), polarization analyzed (GT10-A, Thorlabs), filtered, (ET525/50, Chroma) and finally focused into a gated PMT (H7421-40, Hamamatsu). Data points for scattering patterns were acquired in steps of $5^{\circ}$ from $-90^{\circ}$ to $90^{\circ}$ and an acceptance angle of $3.4^{\circ}$. Typically, measurements settings were $20 \times 2 \mathrm{~s}$ acquisition time and a gate width of $10 \mathrm{~ns}$. To correct for incoherent hyper Rayleigh scattering from the aqueous phase, both the SHS response from the liposome solution and the SHS response from the solution without liposomes are detected under the same conditions. The SHS intensity from the solution is then subtracted from the SHS intensity from the liposome solution. The obtained difference is then normalized to the isotropic SSS signal of pure water, so that we correct for any form of aberration 
due to differences in the beam profile and obtain a value that can be compared to any other measurement done in the same procedure. The reproducibility of the SHS measurements is 1-2 \% (for aqueous solutions) and 5-6\% for the used liposome solutions. The latter number reflects the uncertainty in the reproducibility of the preparation of the liposomes.

$$
\mathrm{S}(\theta)=\left[\frac{I(\theta)_{\text {SHS,liposomes, } P P P}-I(\theta)_{\text {SHS, solution, } P P P}}{I(\theta)_{\text {SHS, water,SSS }}}\right]
$$

All measurements were performed at $25^{\circ} \mathrm{C}$.

\section{S2. Normalization and correction for the difference in size distribution}

In order to get a comparable value for the scattered SHS or SFS light from different samples we normalized the above measured value in Eq. (1) by the number of droplets $\left(N_{d}\right)$ or liposomes $\left(N_{\text {lip }}\right)$, obtainable from the amount of lipid used and the DLS distribution and correct for the difference in the radius $(R)$.

For a monodisperse solution of droplets or liposomes that are smaller than $\sim 200 \mathrm{~nm}$ in radius the total scattered signal $(S)$ from a solution with $N_{p}$ particles that each scatter an intensity $I(\theta)$ scales as follows ${ }^{5}$ :

$$
S(\theta)=I(\theta) N_{p} \propto \alpha(\theta) N_{p} R^{6}
$$

The factor $\alpha$ contains all the information about the surface response per droplet / liposome, independent of its size. Thus, if we want to compare the lipid or water response per droplet or liposome we have to compute the following:

$$
\alpha(\theta)=\frac{\mathrm{S}(\theta)}{N_{p} R^{6}}=I_{\text {norm }}(\theta, R),
$$

which is what we have used in this work, and plot on the y-axis in Fig. 1A. Note that for the SFS data we plot the measured spectrum $\left(I_{S F} / I_{\mathbb{R}}\right)$ and then use the procedure outlined in $S 5$ to compute the average asymmetry per liposome in lipid number density using the fitted amplitudes of the symmetric P-O stretch and the symmetric $\mathrm{CH}_{3}$ stretch mode as input. For both the SHS and SFS experiments we correct for polydispersity by replacing the radius $R$ in Eq. (3) with an effective radius $\left(R_{\text {eff }}\right)$. The procedure to calculate $R_{\text {eff }}$ is described below. 


\section{S3. Calculation of an effective radius}

Since all particles contribute in the same way to the overall intensity of any light scattering experiment, we can use the DLS data to compute an effective radius that can be used for SHS and SFS experiments on polydisperse samples. DLS uses the temporal autocorrelation of scattered light to measure an intensity-weighted particle size distribution histogram. The output of such a measurement is a (normalized) distribution $D(R)$, which we will use here to correct the SHS and SFS signal for variations in the droplet / liposome size distribution, which affects both the scattered intensity per scatterer and the number of scatterers. In other words, we want to replace the total DLS intensity from a polydisperse distribution $\sum_{i} I\left(R_{i}\right)$ by an intensity $I_{\text {norm }}\left(\theta, R_{\text {eff }}\right)$ from a 'monodisperse' solution. The obtained effective radius can then be used to normalize the $\mathrm{SH}$ intensity according to Eq. (3). In this way, we exclude intensity differences based on different sample sizes and obtain the surface scattering contribution $\alpha(\theta)$, which contains information about surface packing, hydration and orientation of water molecules of a single scatterer. Explicitly we have from Eq. (3):

$$
\alpha(\theta)=\frac{\sum_{i} I_{i}(\theta)}{N_{p} R^{6}}=\frac{S(\theta)}{N_{p} R_{e f f}^{6}}=I_{\text {norm }}(\theta)
$$

In the RGD limit, which is applicable here ${ }^{6}$, the intensity of scattered light in a DLS measurement also scales with $R^{6}$, so that:

$$
D(R)=\frac{P(R) R^{6}}{\int P(R) R^{6} d R}
$$

The particle size distribution $P(R)$ is a normalized probability distribution, such that $\int P(R) d R=1$. We can calculate the particle size distribution from the DLS intensity-weighted distribution by:

$$
P(R)=\frac{D(R)}{R^{6}} / \int \frac{D(R)}{R^{6}} d R
$$

Using the particle size distribution we then calculate the effective radius for the liposomes using the following general expression:

$$
R_{\text {eff }, \text { lip }}=\left[\frac{\int P(R) R^{6} d R}{\int \frac{1}{2} P(R)\left(R^{2}+(R-d)^{2}\right) d R}\right]^{1 / 4},
$$


where the denominator takes into account that with changing radius the number of lipids per liposomes changes too (i.e. size and number density are related). For nanodroplets we have the following expression:

$$
R_{e f f, d}=\left[\frac{\int P(R) R^{6} d R}{\int P(R) R^{3} d R}\right]^{1 / 3},
$$

where the denominator is now representing a sphere rather than a hollow shell.

\section{S4. SFS spectral fitting}

The SFS signal (S) can be described by the following Lorentzian line shape expression?:

$$
S_{S F S}(\theta, \omega) \propto\left|A_{N R}(\theta) f(\omega) e^{i \varphi_{N R}}+\sum_{i} \frac{A_{i}(\theta) \gamma_{i}}{\omega-\omega_{i}+i \gamma_{i}}\right|^{2},
$$

where $A_{N R}(\theta)$ is the amplitude and $f(\omega)$ is the spectral shape of a weakly dispersive ('nonresonant') background, $\varphi_{N R}$ is the phase of the background signal relative to that of the resonant signal, $A_{i}(\theta)$ is the amplitude of the $i$ th vibrational mode with the resonance frequency $\omega_{i}$ and linewidth $\gamma_{i}$. The strength of the vibrational mode is proportional to $A_{i}\left(\omega=\omega_{i}\right)$. The SFS spectra $\left(\mathrm{I}_{\mathrm{SF}} / \mathrm{I}_{\mathrm{IR}}\right)$ were fitted using Eq. 9, employing IGOR Pro 6 (WaveMetrics) and using LevenbergMarquardt iterations. The fitted parameters for the SFS spectra are shown in Table S2 and S3. The SFS intensity in the $\mathrm{s}-\mathrm{PO}_{2}{ }^{-}$stretch region in the SPS polarization combination was too low to reliably fit for all the samples.

The SFS spectra that do not show any detectable features are fitted with a $3^{\text {rd }}$ order polynomial.

Table S2: Fitted frequency, amplitude and linewidth for the SFS spectrum in the C-H and P-O stretch region of $\mathrm{d}_{66^{-}}$ DOPC:DPPS liposomes.

\begin{tabular}{|c|ccc|}
\hline mode & $\omega_{i}\left(\mathrm{~cm}^{-1}\right)$ & $\Upsilon_{i}\left(\mathrm{~cm}^{-1}\right)$ & $\mathrm{A}_{\mathrm{i}}$ \\
\hline $\mathrm{r}^{+}$ & 2868 & 20 & 0.15 \\
$\mathrm{~d}_{\mathrm{FR}}^{+}$ & 2926 & 16 & 0.11 \\
$\mathrm{r}^{-}$ & 2968 & 20 & 0.14 \\
$\mathrm{~d}^{-}$ & 2906 & 20 & 0.02 \\
\hline
\end{tabular}


Table S3: Fitted frequency, amplitude and linewidth for the SFS spectra in the P-O stretch.

\begin{tabular}{|cc|cc|cc|c|c|}
\hline \multicolumn{2}{|c|}{ Sample } & \multicolumn{2}{|c|}{ DOPC:DPPS } & \multicolumn{2}{c|}{ DPPC on oil } & DEPC on oil & DOPS:DPPS \\
\hline \multicolumn{2}{|c|}{ Polarization } & SSP & PPP & SSP & PPP & SSP & SSP \\
\hline \multirow{2}{*}{$\mathrm{SS}^{2}$} & $\mathrm{~A}_{\mathrm{i}}$ & 1.13 & 1 & 1.85 & 1 & 1 & 1 \\
$\mathrm{PO}_{2}^{-}$ & $\omega_{i}\left(\mathrm{~cm}^{-1}\right)$ & 1079 & 1079 & 1099 & 1096 & 1085 & 1083 \\
& $\Upsilon_{i}\left(\mathrm{~cm}^{-1}\right)$ & 17 & 17 & 10 & 13 & 20 & 13 \\
& $\mathrm{~A}_{\mathrm{i}}$ & & & 1.31 & 0.93 & & \\
$\mathrm{SS}$ & $\omega_{i}\left(\mathrm{~cm}^{-1}\right)$ & & & 1072 & 1066 & & \\
$\mathrm{C}-\mathrm{O}-\mathrm{P}$ & $\Upsilon_{i}\left(\mathrm{~cm}^{-1}\right)$ & & & 20 & 12 & & \\
& & & & & & & \\
\hline
\end{tabular}

\section{S5. Calculation of the orientational distribution of phosphate groups}

The orientational analysis to calculate the ratio of SFS amplitudes in SSP and PPP polarization combinations of the $\mathrm{s}-\mathrm{PO}_{2}{ }^{-}$vibration is adapted from our procedure published earlier ${ }^{8,9}$ based on the Rayleigh-Gans-Debye approximation in combination with nonlinear light scattering theory. We use a tilt angle $\phi$ of the $\mathrm{PO}_{2}^{-}$group with respect to the surface normal and a twist angle $\psi$ of the $\mathrm{PO}_{2}{ }^{-}$ group around its molecular axis. This results in the following relations ${ }^{10}$ between the surface second-order susceptibility $\left(\boldsymbol{X}^{(2)}\right)$ and molecular hyperpolarizability $\left(\boldsymbol{\beta}^{(2)}\right)$ tensor elements:

$$
\begin{gathered}
\chi_{x x z}^{(2)}=\chi_{y y z}^{(2)}=\frac{1}{2} N\left(\beta_{a a c}^{(2)} \cos ^{2} \psi+\beta_{b b c}^{(2)} \sin ^{2} \psi+\beta_{c c c}^{(2)}\right) \cos \phi+\frac{1}{2} N\left(\beta_{a a c}^{(2)} \sin ^{2} \psi+\beta_{b b c}^{(2)} \cos ^{2} \psi-\beta_{c c c}^{(2)}\right) \cos ^{3} \phi \\
\chi_{z z z}^{(2)}=N\left(\beta_{a a c}^{(2)} \sin ^{2} \psi+\beta_{b b c}^{(2)} \cos ^{2} \psi\right) \cos \phi-N\left(\beta_{a a c}^{(2)} \sin ^{2} \psi+\beta_{b b c}^{(2)} \cos ^{2} \psi-\beta_{c c c}^{(2)}\right) \cos ^{3} \phi
\end{gathered}
$$

with $\mathrm{N}$ the surface density of $\mathrm{PO}_{2}^{-}$groups. We assume that the droplet/liposome interface is azimuthally isotropic. The values of the second-order hyperopolarizability tensor elements were taken from Ref. $^{10}$.

\section{S6. Calculation of the degree of asymmetry based on geometrical arguments}

The number of lipids per leaflet can be calculated assuming that the liposomes have a spherical shape. We assume that each lipid headgroup occupies a constant area, $a$, which is the same at the inner and outer leaflets. ${ }^{11}$ Then we get for the respective number difference $(\Delta \mathrm{N})$ between the outer leaflet and inner leaflet: $\Delta N=\frac{4 \pi\left(\left(R^{2}-(R-d)^{2}\right)\right.}{a} . \Delta N$ can be expressed as a percentage of the total lipid number density per liposome $\left(\mathrm{N}_{\mathrm{tot}}\right)$, which is given by $N_{\text {tot }}=\frac{4 \pi\left(\left(R^{2}+(R-d)^{2}\right)\right.}{a}$. Here, $R$ is the outer radius of the liposome and $d$ is the membrane thickness, for which we take $d \sim 5 \mathrm{~nm}$. 


\section{S7. Calculation of the degree of asymmetry from the SFS data}

For a monodisperse solution, the number densities $\left(N_{d}\right)$ of nanodroplets can be calculated by dividing the volume concentration of oil in the sample $\left(V_{\text {oil }}\right)$ by the volume of one droplet with radius $R_{d}$ :

$$
N_{d}=\frac{V_{\text {oil }}}{\frac{4}{3} \pi R_{d}^{3}} .
$$

For liposomes we have a spherical bilayer rather than a sphere with radius $R_{\text {lip }}$ and thickness $d$ and lipid volume concentration $V_{\text {lip }}$, so that the number density of liposomes is different:

$$
N_{\text {lip }}=\frac{V_{\text {lip }}}{\frac{4}{3} \pi\left(R_{\text {lip }}^{3}-\left(R_{\text {lip }}-d\right)^{3}\right)} .
$$

For the calculation of the number density of liposomes we use $d=5 \mathrm{~nm}^{12}$.

To extract the degree of asymmetry for a certain vibrational mode accounting for polydispersity, we fit the obtained spectra according to Eq. 9 and use the obtained amplitude $A_{i}(\theta)$ in the expression for $\alpha$ (Eq. 4):

$$
\alpha_{l i p, i}\left(\theta, R_{e f f, l i p}\right)=\frac{\left|A_{i}(\theta)\right|^{2}}{N_{l i p} R_{e f f, l i p}^{6}}
$$

This value is now independent of liposomes size, has been corrected for polydispersity and can be compared to other samples. For droplets we obtain the same expression. The parameters of the emulsions and liposomes and calculated degree of asymmetry are shown in Tables S4 and S5.

Table S4: Calculation of the degree of transmembrane asymmetry for $\mathrm{d}_{66}$-DOPC:DPPS liposomes, using a DPPC monolayer on an oil droplet with known lipid density as a reference.

\begin{tabular}{|c|c|c|}
\hline Sample & $\begin{array}{c}\text { DPPC on } \mathrm{C}_{16} \mathrm{D}_{34} \\
\text { (droplets) }\end{array}$ & $\begin{array}{c}\mathrm{d}_{66} \text {-DOPC:DPPS } \\
\text { (liposomes) }\end{array}$ \\
\hline $\mathrm{R}_{\text {eff, }} \mathrm{nm}$ & 97.6 & 41.4 \\
\hline concentration & $2 \mathrm{vol} \%$ & $5 \mathrm{mg} / \mathrm{ml}$ \\
\hline $\mathrm{A}_{\mathrm{i}}, \mathrm{s}-\mathrm{CH}_{3}$ mode & 3.87 & 0.15 \\
\hline$\frac{n_{\text {sample }}}{n_{d}}, \mathrm{~s}-\mathrm{CH}_{3}$ mode & 1 & 0.16 \\
\hline
\end{tabular}

Table S5: Calculation of the degree of head group orientation asymmetry for DOPC:DPPS and DOPS:DPPS liposomes, using a DPPC monolayer on an oil droplet with known lipid density as a reference.

\begin{tabular}{|c|c|c|}
\hline Sample & $\mathrm{R}_{\text {eff }}, \mathrm{nm}$ & $\frac{n_{\text {sample }}}{n_{d}}, \mathrm{~s}-\mathrm{PO}_{2}{ }^{-}$mode \\
\hline DOPC:DPPS & 33 & 0.95 \\
\hline dDOPC:DPPS & 44 & 0.88 \\
\hline DOPS:DPPS & 33 & 0.92 \\
\hline
\end{tabular}




\section{S8. Additional SFS experiments}

In addition to the data shown in the manuscript, we tested all DO/DP tail combinations of PC:PS mixed liposomes. The SFS spectrum of DOPC:DPPS and DOPS:DPPC (Figure S2 and Figure 4) along with DPPC:DPPS and DOPC:DOPS (Figure S2) mixed liposomes were measured. Only the DOPC:DPPS liposomes generate a detectable SFS response in the phosphate spectral region.

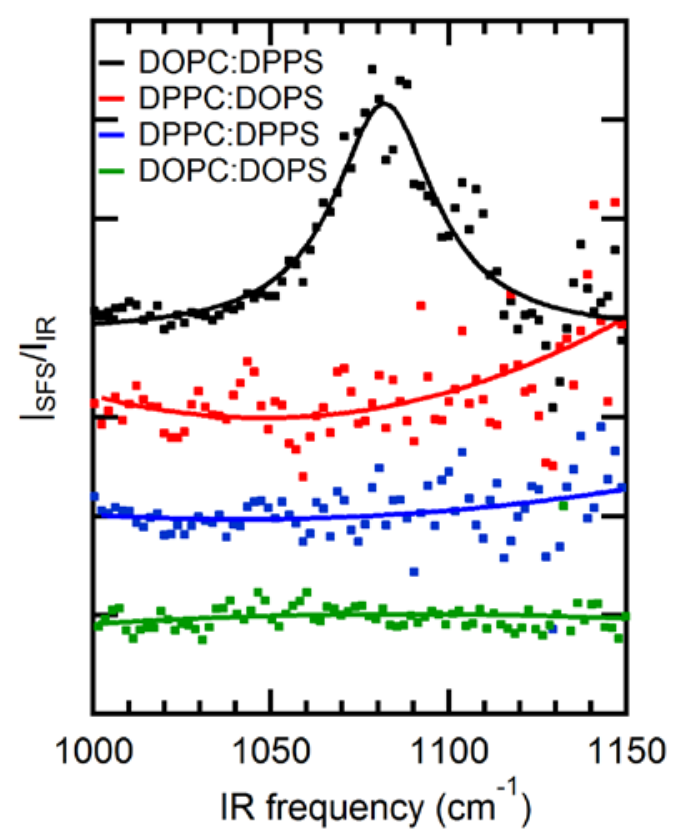

Figure S2. SFS spectra of mixtures of PC and PS lipids with different combinations of fatty acid tails: DOPC:DPPS (black), DPPC:DOPS (red), DPPC:DPPS (blue) and DOPC:DOPS (green). The SFS data are offset vertically for clarity.

\section{References}

(1) de Aguiar, H. B.; Samson, J.-S.; Roke, S. Chem. Phys. Lett. 2011, 512, 76.

(2) de Aguiar, H. B.; Scheu, R.; Jena, K. C.; de Beer, A. G. F.; Roke, S. Phys. Chem. Chem. Phys. 2012, 14, 6826.

(3) Scheu, R.; Chen, Y.; Subinya, M.; Roke, S. J. Am. Chem. Soc. 2013, 135, 19330.

(4) Gomopoulos, N.; Luetgebaucks, C.; Sun, Q.; Roke, S. Opt. Express 2013, $21,815$.

(5) de Beer, A. G. F.; Roke, S. Phys. Rev. B 2009, 79, 155420.

(6) de Beer, A. G. F.; Roke, S. Phys. Rev. B 2007, 75, 245438.

(7) de Aguiar, H. B.; Strader, M. L.; de Beer, A. G. F.; Roke, S. J Phys Chem B 2011, 115, 2970.

(8) Chen, Y.; Jena, K. C.; Lütgebaucks, C.; Okur, H. I.; Roke, S. Nano Lett. 2015, 15, 5558.

(9) de Beer, A. G. F.; Roke, S. J. Chem. Phys. 2010, 132, 234702.

(10) Casillas-Ituarte, N. N.; Chen, X.; Castada, H.; Allen, H. C. J Phys Chem B 2010, 114, 9485.

(11) Israelachvili, J. N. Intermolecular and surface forces; Academic Press; New York, 1991.

(12) Gramse, G.; Dols-Perez, A.; Edwards, M. A.; Fumagalli, L.; Gomila, G. Biophys. J. 2013, 104, 